\title{
The Efficiency of Some Tactical Combinations for the Forms of Rally Directing for World Ranked Tennis Players
}

\author{
Yasser Kamal Mahmoud Salem Ghoneim*
}

This study aim was to determine the prevalence of distinct groundstrokes combinations in 2,3 and 4 stroke rallies in top level tennis. The sample of 39 tennis games in US open 2010, Toronto 2010 and Monte Carlo 2010 with 2054 tactical combinations was analyzed. All rallies were categorized into 2, 3 and 4 groundstrokes combinations with several distinct rally-patterns identified. After applying the proper statistical processing the researcher was able to determine the most effective tactical combinations that ended in winning points which is the main goal from applying all the technical and tactical abilities of any athlete. In conclusion, tennis players should be trained to end the rally within 2 or 3 consecutive strokes as these forms were the most prevalent at the top level.

\section{Introduction}

$\mathbf{T}$ actic is the process of choosing a specific technical skill to be applied in a specific situation using mental abilities according to the changing nature of performance during the game. $(1 \leqslant: 13,14)$

Tennis tactics (2010) mean many different things to different players. (1 $r$ ).Johnson et al (1993) stated that predicting all the possible tactical situations in tennis is the most important tactical rule. The player has to have his own tactical tools that he uses during the game only if he masters his skills and strokes. $(11: 74,89)$. Cooper (2011) also stated that one of the marks of an experienced tennis competitor is thinking beyond the shot you are about to hit. Your odds of hitting a winner are far greater if you set yourself up for it than if you try to create it spontaneously out of sheer shot-making brilliance. (9)..Cahill (2007) mentioned that a good quality rally ball is one that does not give the opponent the opportunity to attack. This ball should be rising up past the baseline when the opponent contacts the ball. If you watch the position of the bounce of top players the ball is not always landing close to the baseline. A ball played with good effect can stop the opponent from attacking even when it is just past the service line on the court.(7).

*Assistant Professor, Sports Games Training Department, The Faculty of Physical Education, Abu Qir, Alexandria University, Egypt.
Stroke combinations during rallies play a major role in tennis tactics. As such, it is of importance to gather knowledge of stroke combination- patterns in top-level tennis players. A rally in tennis is a sequence of shots that starts with serve until the point is won by one of the players $(8,10)$

A groundstroke in tennis is a forehand or backhand shot that is executed after the ball bounces once on the court. The percentage of ground strokes that are used in tennis games reaches over $70 \%$ of the total strokes which increases its importance in winning points. $(10$ : 168)

\section{Ground strokes can be classified into:}

- Down the line shot

- Cross court shot

- Down the middle shot (2:80) (4:67) (11:67)

Identifying stroke-combinations that are used in rallies and their efficiency would be a basic pillar in the training process. Strokecombination can lead to one of the following:

1. Winning a direct point with successful deception using a ground stroke to the wrong opponent's foot

2. Winning a direct point without deception using a winner shot 
3. Winning an indirect point with a wrong opponent's return

4. Winning a point through an opponent's unforced error

Algammal et al (1996) stated that planning for tennis games needs a lot of basic information

that is derived from analyzing performance which includes all types of strokes, errors and efficiency

Assistant Professor, Sports Games Training Department, Faculty of Physical Education Abu Qir - Alexandria University

rates that has to be considered to allow the player to identify ones strengths and weaknesses. (1:194)

Tiley (2010) stated that each player should understand the difference between creating unforced errors compared to forced errors. Unforced errors: mistakes made with no applied pressure from your opponent Forced errors: loss of a point when pressure has been applied from your opponent. Pressure can be created by taking away time and space from your opponent. A player should look to reduce the numbers of unforced errors made and increase the number of forced errors of the opponent.(3).

Wadiea (1994) reasoned that splitting the motor performance and tactics is too difficult as they are connected strongly to form the final decision of how the player reacts in the game and also how he evaluates his opponent's reaction successfully. (5:74)

Murphy (1988), Brown (1989) and Early (1995) as well as USTA (1998) agreed that ground strokes are classified into Forehand and Backhand ground strokes. (2) (10) (6) (13)

\section{Research procedural terminology:}

Rally ending form: is the final outcome for exchanging ground strokes in different playingsituations

The ground stroke-combination: is the series of consecutive groundstrokes that might be similar in skill acquisition or the form of rally ending that results in winning a direct or indirect point.

\section{Research Aim:}

Identifying the efficiency of some tactical combinations for the forms of rally directing for world ranked tennis players

\section{Research thesis:}

What is the prevalence of distinct ground strokes combinations in 2, 3 and 4 ground strokes-rallies?

\section{Research procedures:}

\section{Research method:}

The researcher used the descriptive survey method to perform this research

\section{Research sample:}

The 39 tennis games, all including top 50 tennis players in US open 2010, Toronto 2010 and Monte Carlo 2010, with overall 2054 tactical combinations, were analyzed. Of all, 797 combinations were with 2, 798 combinations with 3 and 450 combinations with 4 consecutive groundstrokes.

. The research was conducted from June 2010 up to January 2011.

\section{Research tools:}

A desk top PC that was connected to a 19 inch LCD using Windows Media player software operating under Windows 7, was used for game observation and to analyze stroke patterns during the rallies. The research was performed in the Lab department in the faculty of physical education for men - AlexandriaUniversity Egypt. In addition, a questionnaire has been used to register the research data to facilitate the statistical processing.

\section{Questionnaire coefficients of Consistency and objectivity pilot studies:}

The content of the questionnaire was presented to a group of tennis experts to confirm the virtual consistency.

The proposed questionnaire then was applied on 100 case of tactical combinations "outside the research sample of combinations" then after 10 days the questionnaire was re-applied on the same sample with the same terms and conditions where the statistical processing was applied using arithmetic mean, standard 
deviation and relation coefficient that was (0.93) which confirmed the consistency of the questionnaire.

The objectivity was proved through giving the questionnaire to an individual who has a good experience in tennis coaching to be applied on the same sample and training him on how to Data collection:

The attacker's position was defined with 3 distinct areas and the areas of the balls contact extract the required information using the research tools. Then statistical processing was applied on his results using Pearson's simple coefficient between the 2 results which was (0.85) that confirmed the objectivity of the questionnaire.

with ground into the opponent's side were divided into a 6 areas as shown in Fig (1).

Figure (1)

\begin{tabular}{|c|c|c|c|c|c|}
\hline 2 & 4 & 6 & 8 & & $\begin{array}{c}\text { Right Side } \\
\text { Spot 1 }\end{array}$ \\
\hline 16 & 14 & 12 & 10 & & $\begin{array}{c}\text { Middle Side } \\
\text { Spot 2 }\end{array}$ \\
\hline 15 & 13 & 11 & 9 & & $\begin{array}{c}\text { Left Side } \\
\text { Spot 3 }\end{array}$ \\
\hline 1 & 3 & 5 & 7 & & \\
\hline
\end{tabular}

Spots for Directing TheBall

Areas of Attack

areas of attack and spots of directing the ball into the opponent's side

In case of the presence of the attacker in spot (1) then spots (2-4-6-8) are down the line and spots (1-3-5-7) are cross courts and spots (11-12-1314-15-16) are down the middle. In case of the presence of the attacker in spot (1) then spots
(2-4-6-8) are down the line and spots (1-3-5-7) are cross courts and spots (11-12-13-14-15-16) are down the middle if the attacker strikes from the right half of the court and vice versa.
Efficiency levels were determined as follows: 1)Winner with deception: 4 degrees, 2) Winner without deception: 3 degrees 3) Indirect point through failed return: 2 degrees 4) A point through unforced error: 1 degree.

\section{Results}

Results of the research are summarized in the following tables: 
Table (1)

\begin{tabular}{|c|c|c|c|c|c|c|c|c|c|c|c|c|c|c|c|c|}
\hline \multirow{4}{*}{$\begin{array}{l}\text { Attacker } \\
\text { presence } \\
\text { spot }\end{array}$} & \multirow{2}{*}{\multicolumn{2}{|c|}{ Strokes forms }} & \multicolumn{12}{|c|}{ Efficiency levels } & \multirow{4}{*}{ 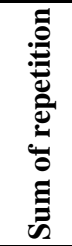 } & \multirow{4}{*}{ 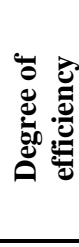 } \\
\hline & & & \multicolumn{3}{|c|}{ Winner with deception } & \multicolumn{3}{|c|}{ Winner without deception } & \multicolumn{3}{|c|}{ Indirect point through failed return } & \multicolumn{3}{|c|}{ A point through unforced error } & & \\
\hline & \multirow{2}{*}{$\stackrel{\overrightarrow{0}}{\stackrel{0}{n}}$} & \multirow{2}{*}{$\begin{array}{l}\vec{\circ} \\
\text { है } \\
\vec{\Xi}\end{array}$} & \multirow{2}{*}{$\stackrel{\mathscr{0}}{\ddot{0}}$} & \multicolumn{2}{|c|}{$\operatorname{Rep} \%$} & \multirow{2}{*}{$\stackrel{0}{\frac{0}{2}}$} & \multicolumn{2}{|c|}{ Rep \% } & \multirow{2}{*}{$\stackrel{0}{0}$} & \multicolumn{2}{|c|}{ Rep \% } & \multirow{2}{*}{$\stackrel{0}{\ddot{0}}$} & \multicolumn{2}{|c|}{$\operatorname{Rep} \%$} & & \\
\hline & & & & $\begin{array}{c}\text { For } \\
\text { column }\end{array}$ & For row & & $\begin{array}{c}\text { For } \\
\text { column }\end{array}$ & For row & & $\begin{array}{c}\text { For } \\
\text { column }\end{array}$ & For row & & $\begin{array}{c}\text { For } \\
\text { column }\end{array}$ & For row & & \\
\hline \multirow{7}{*}{ 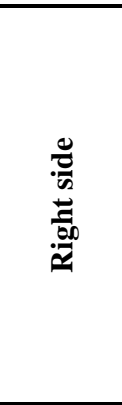 } & $\begin{array}{l}\text { Cross } \\
\text { court }\end{array}$ & $\begin{array}{l}\text { Cross } \\
\text { court }\end{array}$ & - & - & - & r. & $r \leqslant, 0$ & $1 \ldots$ & - & - & - & - & - & - & $r$. & $r$ \\
\hline & $\begin{array}{l}\text { Cross } \\
\text { court } \\
\end{array}$ & $\begin{array}{c}\begin{array}{c}\text { Down the } \\
\text { line }\end{array} \\
\end{array}$ & - & - & - & rs & 10,0 & $\Pi, r$ & ir & $r \wedge, \bullet$ & $19, r$ & ir & $1 \leqslant, Y$ & $19, r$ & it & $r, \xi$ \\
\hline & $\begin{array}{c}\begin{array}{c}\text { Down } \\
\text { the line }\end{array} \\
\end{array}$ & $\begin{array}{c}\begin{array}{c}\text { Down the } \\
\text { line }\end{array} \\
\end{array}$ & - & - & - & - & - & - & - & & - & $\varepsilon$ & $\varepsilon \vee, \uparrow$ & $1 \cdots$ & $\varepsilon$. & 1 \\
\hline & $\begin{array}{c}\begin{array}{c}\text { Down } \\
\text { the line }\end{array} \\
\end{array}$ & $\begin{array}{l}\text { Cross } \\
\text { court } \\
\end{array}$ & - & - & - & - & - & - & r. & $\leq V, T$ & $1 \ldots$ & - & - & - & r. & r \\
\hline & $\begin{array}{l}\text { Cross } \\
\text { court }\end{array}$ & $\begin{array}{c}\begin{array}{c}\text { Down the } \\
\text { middle }\end{array} \\
\end{array}$ & - & - & - & - & - & - & $\bullet$ & 11,9 & $19, r$ & r & ro & $\Lambda \cdot, \mathrm{V}$ & rq & ', \\
\hline & $\begin{array}{c}\begin{array}{c}\text { Down } \\
\text { the }\end{array} \\
\end{array}$ & $\begin{array}{c}\begin{array}{c}\text { Down the } \\
\text { middle }\end{array} \\
\end{array}$ & - & - & - & - & - & - & $\bullet$ & 11,9 & $r, r$ & 11 & ${ }^{1 T}, r$ & $\gg \wedge, \wedge$ & 17 & $1, r$ \\
\hline & \multicolumn{2}{|c|}{ Sum of reps } & - & - & - & 01 & - & - & $\varepsilon r$ & - & - & $\Lambda \leq$ & - & - & $11 \varepsilon$ & - \\
\hline \multirow{9}{*}{ 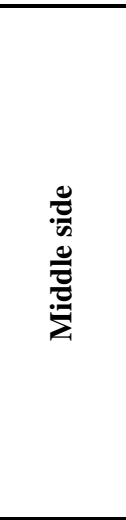 } & $\begin{array}{l}\text { Cross } \\
\text { court }\end{array}$ & $\begin{array}{l}\text { Cross } \\
\text { court } \\
\end{array}$ & $r \varepsilon$ & $\Delta r, r$ & $\leqslant \wedge$ & - & - & - & - & - & - & rq & 9,7 & or & $\circ$. & $\begin{array}{c}r, \xi \\
G\end{array}$ \\
\hline & $\begin{array}{l}\text { Cross } \\
\text { court }\end{array}$ & $\begin{array}{c}\text { Down the } \\
\text { line }\end{array}$ & - & - & - & 01 & $r r, 1$ & $\varepsilon r, \tau$ & 11 & $\wedge, \wedge$ & $9, \xi$ & $\Delta$ & $r \cdot, \varepsilon$ & $\varepsilon V$ & $11 \mathrm{~V}$ & $\frac{1,9}{1,9}$ \\
\hline & $\begin{array}{c}\text { Down } \\
\text { the line }\end{array}$ & $\begin{array}{c}\text { Down the } \\
\text { line }\end{array}$ & r1 & $\leq 7, \vee$ & $r \cdot, o$ & « & $r 0, r$ & $\varepsilon \cdot, r$ & 0 & $\varepsilon$ & $\varepsilon, 9$ & ro & ir & $r \leqslant, \varepsilon$ & $1 \cdot r$ & $\begin{array}{c}r, q \\
v \\
y\end{array}$ \\
\hline & $\begin{array}{c}\begin{array}{c}\text { Down } \\
\text { the line }\end{array} \\
\end{array}$ & $\begin{array}{l}\text { Cross } \\
\text { court } \\
\end{array}$ & - & - & - & $r 4$ & $r r, r$ & $r \cdot, \cdot 0$ & 00 & $\varepsilon \leqslant, r$ & $\leq 7,7$ & rv & 1. & $r r, q$ & 111 & $\stackrel{r}{r}$, \\
\hline & $\begin{array}{l}\text { Cross } \\
\text { court }\end{array}$ & $\begin{array}{c}\begin{array}{c}\text { Down the } \\
\text { middle }\end{array} \\
\end{array}$ & - & - & - & - & - & - & 9 & $v, r$ & $r \leqslant, 7$ & iv & ${ }^{7}, r$ & 10,8 & rq & , \\
\hline & $\begin{array}{c}\text { Down } \\
\text { the }\end{array}$ & $\begin{array}{c}\text { Down the } \\
\text { middle }\end{array}$ & - & - & - & - & - & - & 17 & ir & $r \wedge, 0$ & $\varepsilon$ & $1 \leqslant, \wedge$ & $v_{1}, 0$ & 04 & $\begin{array}{c}1, r \\
a\end{array}$ \\
\hline & $\begin{array}{c}\text { Down } \\
\text { the }\end{array}$ & $\begin{array}{l}\text { Cross } \\
\text { court } \\
\end{array}$ & - & - & - & $\wedge$ & 0,1 & ir & $\varepsilon$ & $r, r$ & 9 & $\Delta$ & $r \cdot, \varepsilon$ & $A r$ & iv & $1, r$ \\
\hline & $\begin{array}{c}\begin{array}{c}\text { Down } \\
\text { the }\end{array} \\
\end{array}$ & $\begin{array}{c}\text { Down the } \\
\text { line }\end{array}$ & - & - & - & rr & $1 \leqslant, 0$ & $r v, 1$ & $r \varepsilon$ & 19,8 & $r \wedge, \vee$ & 10 & 0,0 & $r \leqslant, r$ & Tr & $r$ \\
\hline & \multicolumn{2}{|c|}{ Sum of reps } & $\leqslant 0$ & - & 109 & - & - & Irs & - & - & rv. & - & - & $\Delta 91$ & - & - \\
\hline \multirow{2}{*}{ Left side } & $\begin{array}{c}\text { Down } \\
\text { the line }\end{array}$ & $\begin{array}{l}\text { Cross } \\
\text { court }\end{array}$ & - & - & - & $\wedge$ & $1 \cdots$ & or,r & v & $1 \cdots$ & - & - & - & & 10 & $\stackrel{r}{r}, 0$ \\
\hline & \multicolumn{2}{|c|}{ Sum of reps } & - & - & $\wedge$ & - & - & $v$ & - & - & - & - & - & 10 & - & - \\
\hline
\end{tabular}

The prevalence (total and relative) and efficiency index for the spots of directing 2 consecutive ground strokes according to the rally 
Table (2)

\begin{tabular}{|c|c|c|c|c|c|c|c|c|c|c|c|c|c|c|c|c|c|}
\hline \multirow{3}{*}{$\begin{array}{c}\text { Attacker } \\
\text { presence } \\
\text { spot }\end{array}$} & \multirow{2}{*}{\multicolumn{3}{|c|}{ Stokes forms }} & \multicolumn{12}{|c|}{ Efficiencv levels } & \multirow{3}{*}{ 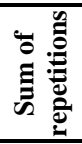 } & \multirow{3}{*}{ 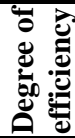 } \\
\hline & & & & \multicolumn{3}{|c|}{ Winner with successful deception } & \multicolumn{3}{|c|}{ Winner without deception } & \multicolumn{3}{|c|}{\begin{tabular}{l|l} 
Indirect point through failed return \\
\end{tabular}} & \multicolumn{3}{|c|}{ A point through unforced error } & & \\
\hline & $\frac{\pi}{5}$ & 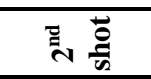 & 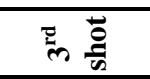 & 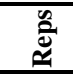 & \multicolumn{2}{|c|}{ Reps \% } & 厸 & \multicolumn{2}{|c|}{ Reps \% } & 啇 & \multicolumn{2}{|c|}{ Reps \% } & 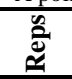 & \multicolumn{2}{|c|}{ Reps \% } & & \\
\hline \multirow{7}{*}{ 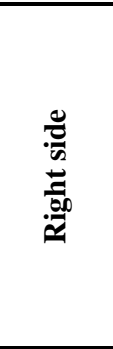 } & Cross court & Cross court & Cross court & - & - & - & - & - & - & $\varepsilon$ & $r$. & $r q, 7$ & 11 & 11,1 & $7 \leqslant, \varepsilon$ & 10 & $1, r V$ \\
\hline & Down the line & Cross court & Down the line & - & - & - & r) & $V Y, \xi$ & $v v, v$ & 7 & r. & $r+, r$ & - & - & - & rv & $r, \mathrm{r}$ \\
\hline & Cross court & $\begin{array}{c}\begin{array}{c}\text { Down the } \\
\text { middle }\end{array} \\
\end{array}$ & Cross court & - & - & - & - & - & - & - & - & - & r. & $r \cdot, r$ & $1 \cdots$ & r. & 1 \\
\hline & Cross court & Down the line & Down the line & 9 & $1 \cdots$ & or & $\wedge$ & $r v, 7$ & $\leqslant V$ & - & - & - & - & - & - & iv & $r, o r$ \\
\hline & $\begin{array}{l}\text { Down the } \\
\text { middle }\end{array}$ & $\begin{array}{c}\begin{array}{c}\text { Down the } \\
\text { middle }\end{array} \\
\end{array}$ & Down the line & - & - & - & - & - & - & 1. & 0. & 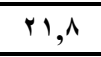 & r & $r, \varepsilon$ & $\vee \wedge, \wedge$ & $\leq 4$ & $1, r Y$ \\
\hline & $\begin{array}{l}\text { Down the } \\
\text { middle }\end{array}$ & Down the line & Cross court & - & - & - & - & - & - & - & - & - & rr & $r r, r$ & $1 \cdots$ & rr & 1 \\
\hline & \multicolumn{3}{|c|}{ Sum of repetitions } & 9 & - & - & rq & - & - & r. & - & - & 99 & - & - & $10 \mathrm{~V}$ & - \\
\hline \multirow{17}{*}{ 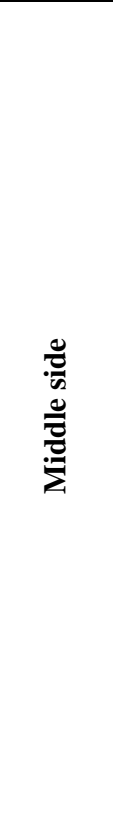 } & Cross court & Cross court & Cross court & - & - & - & $\varepsilon$ & $r, r$ & ro & $\varepsilon$ & 0 & ro & $\wedge$ & $r, r$ & 0 . & 17 & 1.10 \\
\hline & $\begin{array}{l}\text { Down the } \\
\text { middle }\end{array}$ & Cross court & Cross court & - & - & - & ri & ir & $01, Y$ & 1. & 14,0 & $r \leqslant, \varepsilon$ & 1. & $r, q$ & $r \leqslant, \varepsilon$ & \&1 & $r, r v$ \\
\hline & $\begin{array}{l}\text { Down the } \\
\text { middle }\end{array}$ & Cross court & Down the line & - & - & - & rq & $1 \leqslant, 9$ & $r q, 7$ & 19 & $r r, v$ & $r q, \wedge$ & rq & $v, r$ & $m, 7$ & vi & $r$ \\
\hline & $\begin{array}{l}\text { Down the } \\
\text { middle }\end{array}$ & Down the line & Cross court & - & - & - & $\leq 4$ & $r q, r$ & $1 \ldots$ & - & - & - & - & - & - & $\leqslant 4$ & $r$ \\
\hline & $\begin{array}{l}\text { Down the } \\
\text { middle }\end{array}$ & Down the line & Down the line & r. & $1 \ldots$ & $r r, \Lambda$ & $\leqslant 1$ & $r r, \varepsilon$ & $i v, r$ & - & - & - & - & - & - & 71 & $r, r r$ \\
\hline & $\begin{array}{l}\text { Down the } \\
\text { middle }\end{array}$ & Down the line & $\begin{array}{c}\text { Down the } \\
\text { middle }\end{array}$ & - & - & - & 0 & $r, \wedge$ & $1 \cdot, \mathrm{V}$ & $r$. & $r v, o$ & $7 r, 9$ & ir & $r, 0$ & $r 0, \varepsilon$ & $\varepsilon V$ & 1,10 \\
\hline & Cross court & $\begin{array}{l}\text { Down the } \\
\text { middle }\end{array}$ & $\begin{array}{l}\text { Down the } \\
\text { middle }\end{array}$ & - & - & - & - & - & - & - & - & - & ro & $v, r$ & $1 \ldots$ & ro & 1 \\
\hline & Down the line & $\begin{array}{c}\text { Down the } \\
\text { middle }\end{array}$ & $\begin{array}{l}\text { Down the } \\
\text { middle }\end{array}$ & - & - & - & - & - & - & - & - & - & ro & $v, r$ & $1 \ldots$ & ro & 1 \\
\hline & Cross court & Cross court & $\begin{array}{c}\begin{array}{c}\text { Down the } \\
\text { middle }\end{array} \\
\end{array}$ & - & - & - & - & - & - & - & - & - & $r v$ & $1 \cdot, 1$ & $1 \ldots$ & $r v$ & 1 \\
\hline & Cross court & $\begin{array}{l}\text { Down the } \\
\text { middle }\end{array}$ & Down the line & - & - & - & - & - & - & - & - & - & 17 & $\varepsilon, \mathrm{V}$ & $1 \ldots$ & 17 & 1 \\
\hline & Cross court & Down the line & $\begin{array}{c}\text { Down the } \\
\text { middle }\end{array}$ & - & - & - & - & - & - & - & - & - & ri & 7,1 & $1 \ldots$ & ri & 1 \\
\hline & Down the line & $\begin{array}{l}\text { Down the } \\
\text { middle }\end{array}$ & Cross court & - & - & - & - & - & - & - & - & - & ri & 7,1 & $1 \ldots$ & r) & 1 \\
\hline & Down the line & $\begin{array}{c}\text { Down the } \\
\text { middle }\end{array}$ & Down the line & - & - & - & - & - & - & - & - & - & ro & $v, r$ & $1 \cdots$ & ro & 1 \\
\hline & $\begin{array}{c}\text { Down the } \\
\text { middle }\end{array}$ & $\begin{array}{c}\text { Down the } \\
\text { middle }\end{array}$ & $\begin{array}{l}\begin{array}{c}\text { Down the } \\
\text { middle }\end{array} \\
\end{array}$ & - & - & - & - & - & - & - & - & - & rA & 11,1 & $1 \cdots$ & rs & 1 \\
\hline & $\begin{array}{l}\text { Down the } \\
\text { middle }\end{array}$ & $\begin{array}{l}\text { Down the } \\
\text { middle }\end{array}$ & Down the line & - & - & - & 1. & $0, V$ & $r r, r$ & - & - & - & $r_{0}$ & $1 \cdot, r$ & $\vee \vee, \wedge$ & $\leqslant 0$ & $1, \leqslant \leqslant$ \\
\hline & $\begin{array}{c}\text { Down the } \\
\text { middle }\end{array}$ & $\begin{array}{c}\text { Down the } \\
\text { middle }\end{array}$ & Cross court & - & - & - & rr & $1 r, 7$ & $r v, 1$ & iv & $r, r$ & r) & $\leqslant r$ & $1 r, r$ & 01,9 & 11 & $1, v_{0}$ \\
\hline & \multicolumn{3}{|c|}{ Sum of repetitions } & $r$. & - & - & ivo & - & - & $\wedge$. & - & - & $r \leqslant 1$ & - & - & 717 & - \\
\hline \multirow{2}{*}{$\begin{array}{l}\text { Left } \\
\text { side }\end{array}$} & Cross court & Cross court & Cross court & - & - & - & - & - & - & - & - & - & ro & $1 \ldots$ & $1 \ldots$ & ro & 1 \\
\hline & \multicolumn{3}{|c|}{ Sum of repetitions } & - & - & - & - & - & - & - & - & - & - & - & - & ro & - \\
\hline
\end{tabular}

The repetitions and percentage and efficiency degrees for the spots of directing 3 consecutive ground strokes according to the rally 
Table (3)

\begin{tabular}{|c|c|c|c|c|c|c|c|c|c|c|c|c|c|c|c|c|c|c|}
\hline \multirow{4}{*}{$\begin{array}{c}\text { Attacker } \\
\text { presence } \\
\text { spot }\end{array}$} & \multirow{2}{*}{\multicolumn{4}{|c|}{ Stokes forms }} & \multicolumn{12}{|c|}{ Efficiency levels } & \multirow{4}{*}{$\begin{array}{c}\text { Sum } \\
\text { of } \\
\text { repetit } \\
\text { ion }\end{array}$} & \multirow{4}{*}{$\begin{array}{c}\text { Degre } \\
\text { e of } \\
\text { efficie } \\
\text { ncy }\end{array}$} \\
\hline & & & & & \multicolumn{3}{|c|}{ Winner with successful } & \multicolumn{3}{|c|}{ Winner without deception } & \multicolumn{3}{|c|}{ Indirect point through } & \multicolumn{3}{|c|}{ A point through unforced } & & \\
\hline & \multirow{2}{*}{$\underset{\vec{E}}{\vec{E}}$} & \multirow{2}{*}{$\stackrel{\vec{\Xi}}{\frac{\vec{E}}{\omega}}$} & \multirow{2}{*}{$\Xi \frac{\vec{\theta}}{\omega}$} & \multirow{2}{*}{ 辛 $\frac{\vec{E}}{\omega}$} & \multirow{2}{*}{$\stackrel{n}{\dddot{2}}$} & \multicolumn{2}{|c|}{$\operatorname{Rep} \%$} & \multirow{2}{*}{$\stackrel{0}{\ddot{2}}$} & \multicolumn{2}{|c|}{$\operatorname{Rep} \%$} & \multirow{2}{*}{ 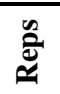 } & \multicolumn{2}{|c|}{ Rep \% } & \multirow{2}{*}{$\stackrel{0}{\ddot{2}}$} & \multicolumn{2}{|c|}{$\operatorname{Rep} \%$} & & \\
\hline & & & & & & $\begin{array}{c}\text { For } \\
\text { column }\end{array}$ & For row & & $\begin{array}{c}\text { For } \\
\text { Cor }\end{array}$ & For row & & $\begin{array}{c}\text { For } \\
\text { column }\end{array}$ & For row & & $\begin{array}{c}\text { For } \\
\text { column }\end{array}$ & For row & & \\
\hline \multirow{5}{*}{ 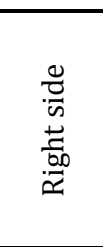 } & $\begin{array}{l}\text { Cross } \\
\text { court }\end{array}$ & $\begin{array}{l}\text { Cross } \\
\text { court }\end{array}$ & $\begin{array}{l}\text { Cross } \\
\text { court }\end{array}$ & $\begin{array}{l}\text { Down the } \\
\text { line }\end{array}$ & - & - & - & - & - & - & 1. & $\varepsilon 0,0$ & - & - & $\varepsilon$ & $77, \mathrm{~V}$ & $r$. & $1, r$ \\
\hline & $\begin{array}{l}\text { Cross } \\
\text { court }\end{array}$ & own the midd & own the midd & $\begin{array}{l}\text { Cross } \\
\text { court }\end{array}$ & - & - & - & 1. & $1 \ldots$ & $r r, \Lambda$ & Ir & $0 \leqslant, 0$ & - & - & $\varepsilon$. & $\varepsilon \vee, T$ & $\varepsilon r$ & $1, \mathrm{VI}$ \\
\hline & $\begin{array}{l}\text { Cross } \\
\text { court }\end{array}$ & \begin{tabular}{|l|} 
Cross \\
court \\
\end{tabular} & $\begin{array}{c}\text { Down the } \\
\text { line }\end{array}$ & $\begin{array}{l}\text { Cross } \\
\text { court }\end{array}$ & 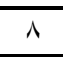 & $1 \ldots$ & $1 \ldots$ & - & - & - & - & - & - & - & - & - & $\wedge$ & $\varepsilon$ \\
\hline & $\begin{array}{l}\text { Cross } \\
\text { court }\end{array}$ & $\begin{array}{l}\text { Cross } \\
\text { court }\end{array}$ & \begin{tabular}{|l|} 
Cross \\
court \\
\end{tabular} & $\begin{array}{l}\text { Cross } \\
\text { court } \\
\end{array}$ & - & - & - & - & - & - & - & - & - & 1. & $r$. & $1 \ldots$ & 1. & 1 \\
\hline & \multicolumn{4}{|c|}{ Sum of reps } & $\Lambda$ & - & - & 1. & - & - & Tr & - & - & 0. & - & - & 9. & - \\
\hline \multirow{16}{*}{ 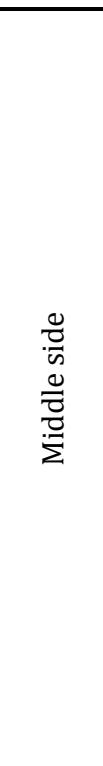 } & $\begin{array}{l}\text { Cross } \\
\text { court }\end{array}$ & own the midd & $\begin{array}{l}\begin{array}{c}\text { Down the } \\
\text { line }\end{array} \\
\end{array}$ & $\begin{array}{l}\text { Cross } \\
\text { court }\end{array}$ & - & - & - & $r$. & $1 r, 9$ & $\pi, 0$ & - & - & - & Ir & $v, v$ & $r v, 0$ & rr & $r, r_{O}$ \\
\hline & own the midd & own the midd & \begin{tabular}{|l|}
$\begin{array}{c}\text { Down the } \\
\text { line }\end{array}$ \\
\end{tabular} & $\begin{array}{l}\text { Cross } \\
\text { court }\end{array}$ & - & - & - & - & - & - & - & - & - & rI & $1 \pi, \varepsilon$ & $1 \ldots$ & r) & 1 \\
\hline & own the midd & own the midd & own the midd & $\begin{array}{l}\text { Cross } \\
\text { court }\end{array}$ & - & - & - & Kr & $1 \leqslant, r$ & 0. & - & - & - & rr & $1 \leqslant, r$ & 0. & $\varepsilon \varepsilon$ & $r$ \\
\hline & \begin{tabular}{|l|}
$\begin{array}{l}\text { Cross } \\
\text { court }\end{array}$ \\
\end{tabular} & \begin{tabular}{|c|}
$\begin{array}{c}\text { Down the } \\
\text { line }\end{array}$ \\
\end{tabular} & \begin{tabular}{|l|}
$\begin{array}{c}\text { Down the } \\
\text { line }\end{array}$ \\
\end{tabular} & $\begin{array}{l}\text { Cross } \\
\text { court }\end{array}$ & - & - & - & & & & - & - & - & 10 & 9,7 & $1 \ldots$ & 10 & 1 \\
\hline & $\begin{array}{l}\text { Down the } \\
\text { line }\end{array}$ & own the midd & $\begin{array}{l}\text { Cross } \\
\text { court }\end{array}$ & $\begin{array}{l}\text { Cross } \\
\text { court }\end{array}$ & - & - & - & rI & $1 \pi, \varepsilon$ & $1 \ldots$ & - & - & - & - & - & - & $r$ & $r$ \\
\hline & $\begin{array}{l}\text { Down the } \\
\text { line }\end{array}$ & \begin{tabular}{|c|}
$\begin{array}{c}\text { Cross } \\
\text { court }\end{array}$ \\
\end{tabular} & \begin{tabular}{|c|} 
court \\
own the midd \\
\end{tabular} & \begin{tabular}{|c|} 
count \\
$\begin{array}{c}\text { Down the } \\
\text { line }\end{array}$ \\
\end{tabular} & - & - & - & 10 & 9,8 & $1 \ldots$ & - & - & - & - & - & - & 10 & $r$ \\
\hline & $\begin{array}{l}\text { Cross } \\
\text { court }\end{array}$ & own the midd & \begin{tabular}{|l|}
$\begin{array}{c}\text { Cross } \\
\text { court }\end{array}$ \\
\end{tabular} & $\begin{array}{l}\text { Cross } \\
\text { court }\end{array}$ & - & - & - & 1. & 7,0 & $1 \ldots$ & - & - & - & - & - & - & 1. & $r$ \\
\hline & $\begin{array}{l}\text { Cross } \\
\text { court }\end{array}$ & \begin{tabular}{l|}
$\begin{array}{l}\text { Cross } \\
\text { court }\end{array}$ \\
\end{tabular} & $\begin{array}{l}\text { Cross } \\
\text { court }\end{array}$ & $\begin{array}{l}\text { Cross } \\
\text { court }\end{array}$ & - & - & - & - & - & - & - & - & - & $r$ & $1 r, \varepsilon$ & $1 \ldots$ & ri & 1 \\
\hline & own the midd & \begin{tabular}{|l|} 
Down the \\
line
\end{tabular} & $\begin{array}{l}\text { Down the } \\
\text { line }\end{array}$ & $\begin{array}{l}\text { Down the } \\
\text { line }\end{array}$ & - & - & - & $r$. & $1 Y, q$ & $1 \ldots$ & - & - & - & - & - & - & $r$. & $r$ \\
\hline & own the midd & \begin{tabular}{|c|}
$\begin{array}{c}\text { Down the } \\
\text { line }\end{array}$ \\
\end{tabular} & $\begin{array}{l}\text { Cross } \\
\text { court } \\
\end{array}$ & $\begin{array}{c}\text { Down the } \\
\text { line }\end{array}$ & - & - & - & - & - & - & - & - & - & 10 & $9, \mathrm{~V}$ & $1 \ldots$ & 10 & 1 \\
\hline & own the midd & own the midd & own the midd & $\begin{array}{c}\begin{array}{c}\text { Down the } \\
\text { line }\end{array} \\
\end{array}$ & - & - & - & - & - & - & - & - & - & $r V$ & $1 V, \varepsilon$ & $1 \cdots$ & TV & 1 \\
\hline & own the midd & \begin{tabular}{|c|}
$\begin{array}{c}\text { Down the } \\
\text { line }\end{array}$ \\
\end{tabular} & \begin{tabular}{|l|} 
own the midd \\
\end{tabular} & \begin{tabular}{|l|} 
Cross \\
court \\
\end{tabular} & - & - & - & rY & $1 \leqslant, Y$ & $1 \ldots$ & - & - & - & - & - & - & $r r$ & $r$ \\
\hline & own the midd & \begin{tabular}{|l|} 
Cross \\
court \\
\end{tabular} & \begin{tabular}{|l|}
$\begin{array}{l}\text { Down the } \\
\text { line }\end{array}$ \\
\end{tabular} & \begin{tabular}{|c|}
$\begin{array}{c}\text { Down the } \\
\text { line }\end{array}$ \\
\end{tabular} & $r$. & $1 \ldots$ & $1 \ldots$ & - & - & - & - & - & - & - & - & - & $r$. & $\varepsilon$ \\
\hline & own the midd & $\begin{array}{l}\text { Cross } \\
\text { court }\end{array}$ & $\begin{array}{l}\text { Cross } \\
\text { court }\end{array}$ & $\begin{array}{l}\begin{array}{l}\text { Cross } \\
\text { court }\end{array} \\
\end{array}$ & - & - & - & 10 & $9, v$ & $r 0, V$ & 0 & $r \Lambda, 0$ & 11,9 & YY & $1 \leqslant, r$ & or, \& & $\varepsilon r$ & $1, \lambda \mu$ \\
\hline & own the midd & own the midd & $\begin{array}{c}\text { Down the } \\
\text { line }\end{array}$ & own the midd & - & - & - & 1. & 7,0 & 00,0 & $\wedge$ & 71,0 & $\leqslant 0,0$ & - & - & - & 11 & $r, 07$ \\
\hline & \multicolumn{4}{|c|}{\begin{tabular}{|c|} 
Sum of reps \\
\end{tabular}} & $r$. & - & - & 100 & - & - & $1 \pi$ & - & - & 100 & - & - & $r \leqslant r$ & - \\
\hline \multirow{2}{*}{$\begin{array}{l}\text { Left } \\
\text { side }\end{array}$} & $\begin{array}{l}\text { Cross } \\
\text { court }\end{array}$ & \begin{tabular}{l|l} 
Down the \\
line
\end{tabular} & own the midd & $\begin{array}{c}\text { Down the } \\
\text { line }\end{array}$ & - & - & - & - & - & - & ir & $1 \cdots$ & $\varepsilon \leqslant, 0$ & 10 & $1 \cdots$ & 00,0 & $r v$ & $1, \leqslant \leqslant$ \\
\hline & & Sum o & ff reps & & - & - & - & - & - & - & - & - & - & 10 & - & - & $r V$ & - \\
\hline
\end{tabular}

The repetitions and percentage and efficiency degrees for the spots of directing \& consecutive ground strokes according to the rally 


\section{Discussion:}

First: The prevalence of 2 groundstrokescombination rallies with attacker in area 1:

\section{1-In case of the attacker's presence in spot 1:}

Table (1) shows that the highest efficiency (3) was obtained for cross court-cross court combination, followed by the cross court- down the line combination with an efficiency degree of 2,44 and 2.42, respectively. Such cross courts shots passes the middle of the net which have the least height which likely provide safer pass, as suggested by Gold (1993). In addition, the direct winner without deception is most prevalent way of winning points for 2 rally groundstrokes combination. The least prevalent (20 repetitions, 34.5\%) was cross court-cross court combination. Winning a point through a failed return had the prevalence, (20 repetitions, $47.6 \%$ ) with down the line-cross court and cross court- down the middle combination being most and least prevalent, respectively..Winning a point through an unforced error is most prevalent (40 repetitions, $47.6 \%$ ) with down the line- down the line and down the middle- down the middle combinations being most and least prevalent, respectively.

\section{2-In case of the attacker's presence in spot 2:}

It is obvious in table (1) that the highest efficiency degree 2.47 was for the 2 consecutive strokes down the line then down the line, followed by cross court then cross court with efficiency degree 2.44. These close values for both combinations can be referred to the trials of the attacker to use deception and direct these strokes against the opponent's movement direction before he regains his balance through returning back to middle of court or trying for the ball. Also it is obvious that a direct winner with deception had the highest repetition of 24 and a percentage of $53.3 \%$ was for cross court then cross court combination and the least was 21 repetitions and a percentage of $46.7 \%$ was for the combination of down the line then down the line. Also in the same table we can see that a winner without deception had the highest repetitions of 51 with a percentage of $32.1 \%$ for the cross court then down the line combination and the least repetitions of 8 with a percentage of $5.1 \%$ for down the middle then cross court combination, also it showed that winning a point through a failed return had the highest repetitions with a percentage of $44.3 \%$ for the combination of down the line then cross court and the least was 4 with a percentage of $3.2 \%$ for the combination of down the middle then cross court. Also winning a point through an unforced error had the highest repetitions of 55 with a percentage of $20.4 \%$ was for the combination of cross court then down the line and the least of 15 repetitions with a percentage of $5.5 \%$ for down the middle then down the line.

\section{3-In case of the attacker's presence in spot 3:}

In table (1) there was only one tactical combination in this spot which is down the line then cross court with an efficiency degree of 2.53 and this can be referred to that cross court ground strokes allows the attacker in this spot to switch the direction of the stroke to the weak side of the opponent on his backhand (USTA, 1998)

Second: The efficiency of tactical forms to end the rally using 3 consecutive ground strokes:

\section{1-In case of the attacker's presence in spot 1:}

Table (2) shows that the highest efficiency degrees 3.53 was for the combination of cross court then down the line then down the line followed by down the line then cross court then down the line with efficiency degree 2.78 as this can be referred to that the attacker was able to deceive his opponent when the attacker predicted that his opponent will run quickly to the middle of the court to put the ground stroke against his movement direction (Gold 1993), this also can be confirmed within the results of the same table as the combination of cross court then down the line then down the line has the 
percentage of $53 \%$ under the category of winning a point with successful deception. Winning a point without deception had 21 repetitions with a percentage of $72.4 \%$ for the combination of down the line then cross court then down the line and the least repetitions of 8 and percentage of $27.6 \%$ was for the combination of cross court then down the line then down the line. Also winning an indirect point with a failed return had the highest repetitions of 10 with percentage of $50 \%$ was for the combination of down the middle then down the middle then down the line and the least was 4 repetitions with percentage $20 \%$ for the combination of cross court then cross court then cross court. Also winning a point with an unforced error had the highest repetitions of 36 and percentage of $36.4 \%$ was for the combination of down the middle then down the middle then down the line and the least with 11 repetitions and percentage of $11.1 \%$ for cross court then cross court then cross court combination.

\section{2-In case of the attacker's presence in spot 2:}

Table (2) shows us that highest efficiency degrees 3.33 was for the combination of down the middle then down the line then down the line followed by down the middle then down the line then cross court combination with an efficiency degree 3 which is so close to the first combination and this can be referred to the attacker's trials to use deception and switch the direction of his ground strokes on the opposite direction of his opponent's movements before he is capable of restoring his balance through coming back to the middle of the court or trying for the far ball especially that down the line strokes are so deep to push the opponent to return to the base line which allows the attacker to return a winning cross court with a very acute angle (Murphy 1988). The same table states that winning a direct point without deception had the highest repetition of 46 and percentage of $26.3 \%$ that was for the combination of down the middle then down the line then cross court and the least was 4 repetitions with percentage of $2.2 \%$ for the combination of cross court then cross court then cross court. Also it is obvious that winning and indirect point through a failed return had the highest repetitions of 30 and percentage of $37.5 \%$ for the combination of down the middle then down the line then down the middle and the least was 4 repetitions and percentage of $5 \%$ for the combination of cross court then cross court then cross court. The same table shows us that winning a point through an unforced error had the highest repetitions of 42 and percentage of $12.3 \%$ was for the combination of down the middle then down the middle then cross court and the least were 8 repetitions and percentage of $2.3 \%$ for the combination of cross court then cross court then cross court.

\section{3-In case of the attacker's presence in spot 3:}

Table (2) shows us that there is only one tactical combination in this playing situation which is cross court then cross court then cross court and the highest efficiency degree for this combination was 1 and this can be referred to that cross court from spot 3 depends of driving the ball to the opponent's back hand which is theoretically the weakest stroke amongst all and it also gives the opponent the chance to attack and force his opponent to commit an unforced error.

Second: The efficiency of tactical forms to end the rally using 4 consecutive ground strokes:

\section{1-In case of the attacker's presence in spot 1:}

Table (3) shows us that the highest efficiency degree of 4 was for the combination of cross court then cross court then down the line then cross court followed by cross court then down the middle then down the middle then cross court with an efficiency degree of 1.76 and this can be referred to that the attacker succeeded in upsetting the balance of his opponent through the deep $3^{\text {rd }}$ stroke followed by a fourth cross 
court away from his position. This combination is considered to be the most difficult as it only was repeated 8 times among 90 ground combinations and it is also confirmed in the percentage of unforced errors that was $47.6 \%$ (Gold 1993). Winning a direct point without deception had the highest repetitions of 10 and a percentage of $100 \%$ was for the combination of cross court the down the middle then down the middle then cross court. Winning a direct point through a failed return had also the highest repetitions of 12 and a percentage of $54.5 \%$ for the combination of cross court the down the middle then down the middle then cross court and the least repetitions of 10 and percentage of $45.5 \%$ was for the combination of cross court then cross court then cross court then down the line. Also winning a point through an unforced opponent's error had the highest repetitions of 20 and percentage of $40 \%$ for the combination of cross court then cross court then cross court then down the line and the least of 10 repetitions and percentage of $20 \%$ for the combination of down the middle then cross court then cross court then cross court.

\section{2-In case of the attacker's presence in spot 2:}

Table (3) shows that the highest efficiency degree of 4 was for the combination of down the middle then cross court then down the line then down the line followed by the combination of down the line then down the middle then cross court then cross court with an efficiency degree of 3 and this can be referred to the trials of the attacker to use deception in playing winners or in directing his ground strokes opposite to the movement direction of his opponent before he is able to restore his balance through coming back to the middle of the court or trying to reach the far ball due to the high depth of down the line ground strokes that push the opponent to go back to the base line after the ball (Murphy 1988). The results in the same table also states that winning with a successful deception had the highest efficiency degree and the winner shot had the highest repetition of 155 out of 34 combinations. Also winning a point through an unforced error had the highest repetition of 27 and percentage of $17.4 \%$ for the combination of down the middle then down the middle then down the middle then down the line and the least of 12 repetitions and percentage of $7.7 \%$ was for the combination of cross court then down the middle then down the line then cross court.

\section{3-In case of the attacker's presence in spot 3:}

Table (3) shows that there is only one tactical combination that is applied when the attacker is playing from spot 3 which is cross court then down the line then down the middle then down the line with efficiency degree of 1.44 and this can be referred to that most of the strokes that are played from this spot depends on the backhand side of the opponent which is theoretically the weakest stroke amongst all and it also gives the opponent the chance to attack and force his opponent to commit an unforced error.

\section{Conclusions:}

In light of the research's sample and procedure, CONCLUSION CAN BE AS FOLLOWS:

1. Concerning 2 groundstrokes rallies, 6,8 and 1 combinations in area 1,2 and 3 were determined, respectively.

2. Concerning the forms of ending the rally with 3 consecutive ground strokes, there was 6 combinations in spot 1 and 16 combinations in spot 2 while there was only 1 combination in spot 3 .

3. Concerning the forms of ending the rally with 4 consecutive ground strokes, there was 4 combinations in spot 1 and 15 combinations in spot 2 while there was only 1 combination in spot 3 .

\section{Recommendations:}

1. The researcher recommends that tennis players should be trained to end the rally 
with 2 or 3 consecutive strokes as these forms were the most frequent among world ranked players games with a total percentage of $77.99 \%$. Rallies with 4 groundstrokes had prevalence rate of modest $22.1 \%$.

2. Groundstrokes should be emphasized in the training as they have large impact on final game outcome.

\section{References:}

1- AbdelnabiAlgammal\& Mohamed Shamoun:

Mental training in tennis, $1^{\text {st }}$ Ed., Dar AlfekrAlarabi, 1996

\section{2- Chet Murphy:}

Advanced Tennis, wm. C. Brown Publishers. U.S.A. 1988.

\section{3- Craig Tiley:}

Stroke and tactical fundamentals in tennis, Tennis Australis, 2010

(http://www.tennis.com.au/wpcontent/uploads/2010/08/Stroke-and-Tactical-

Fundamentals.pdf)

\section{4- Dick Gold:}

Tennis, Anyone, May field Publishing Company Palo/A to, California, 1993.

\section{5- Elaine WadeaFarag:}

Tennis, MoshaatElmaaref, Alexandria, 1994

\section{6- Frank Early:}

Tennis strokes that win, library of congress , 1995.

\section{7- Garry Cahill:}

Rally Ball, International tennis federation, Coaching committee, 2007 (http://www.itftennis.com/shared/medialibrary/p df/original/IO_29145_original.PDF)

\section{8- Jack L. Groppel:}

World - class tennis technique, Human Kinetics Publishers, 2001.

\section{9- Jeff Cooper:}

Five Great Shot Combinations, The New York A. times company, About.com, 2011 (http://tennis.about.com/cs/yourgame/a/shotcom bination.htm)

\section{0- Jim Brown:}

Tennis Step to success, Leisure press, 1989.

\section{1- Johnson I Xantlios:}

Tennis Step to success, Leisure press, 1993.

\section{I r - Tennis Tactics. Com}

Tennis

tactics, 2010

(http://www.besttennistactics.com/\#)

1 $r$ - US Tennis Association:

Coaching Youth Tennis, library of congress , 1998

\section{1 \& - WadieaYassin:}

Training principles in boxing, part 2 , the ministry of scientific research, Iraq, 1983

\section{Yasser K. M. Ghoneim:}

Studying the volume and variation of tennis strokes in Masters championship, Application and theory journal, the faculty of sports education, Alexandria University, 2003 
Yasser Kamal Mahmoud Salem Ghoneim 